\title{
Dynamic Recrystallization and Texture Evolution of GW94 Mg Alloy During Multi- and Unidirectional Impact Forging
}

\author{
S. S. A. Shah ${ }^{1,2} \cdot$ M. G. Jiang ${ }^{1} \cdot$ D. $\mathrm{Wu}^{1} \cdot$ U. Wasi ${ }^{1,3} \cdot$ R. S. Chen ${ }^{1}$ \\ Received: 11 December 2017 / Revised: 22 January 2018/Published online: 25 April 2018 \\ (C) The Chinese Society for Metals and Springer-Verlag GmbH Germany, part of Springer Nature 2018
}

\begin{abstract}
Multi- and unidirectional impact forgings were successfully applied to a (GW94) Mg-RE alloy. The microstructure and texture evolution were investigated systematically. The obtained results indicated that during unidirectional impact forging, a bimodal chain deform microstructure was sustained till last forging pass, whereas $\{10-12\}$ extension twins-assisted continuous dynamic recrystallization took place during the multidirectional impact forging (MDIF). The coalescence and intersection of $\{10-12\}$ extension twins during MDIF efficiently refined the original coarse grains and led to an almost recrystallized homogeneous microstructure. The texture analysis demonstrated that unidirectional impact forging yielded out the strong basal texture; however, MDIF resulted in non-basal texture, which was attributed to the cooperative effects of continuous DRX, twinning, and MDIF itself during the deformation process.
\end{abstract}

Keywords Magnesium alloys $\cdot$ Forging $\cdot$ Twinning $\cdot$ Recrystallization $\cdot$ Texture

\section{Introduction}

Magnesium alloys have attracted significant attentions due to their applications in transportation industries as a lightest structural metallic material, which directly translates to energy saving and environment protection. Effective utilization of magnesium alloys in the automobile industry entails their development with high specific strength.

In recent decades, newly developed $\mathrm{Mg}-\mathrm{Gd}-\mathrm{Y}$ alloys have received extensive interests due to their ultrahigh strength both at room and elevated temperatures [1-3], better creep resistance than WE54 alloy [4] and even being

Available online at http://link.springer.com/journal/40195

D. $\mathrm{Wu}$

dwu@imr.ac.cn

$\triangle$ R. S. Chen

rschen@imr.ac.cn

1 The Group of Magnesium Alloys and Their Applications, Institute of Metal Research, Chinese Academy of Sciences, Shenyang 110016, China

2 University of Chinese Academy of Sciences, Beijing 100049, China

3 School of Materials Science and Engineering, University of Science and Technology of China, Hefei 230026, China better than some conventional $\mathrm{Al}$ alloys [5]. However, most of these researches mainly focus on extrusion $[2,6]$ and rolling processes [7, 8]. About forging process on $\mathrm{Mg}-$ RE alloys, only a few papers could be found, which indicates their bad forgeability during the traditional hydrostatic forging process [9].

Previous studies about multidirectional forging (MDF) were mainly concerned with steel [10], aluminum alloys [11], and some of the $\mathrm{Mg}-\mathrm{Al}-\mathrm{Zn}$ alloys [12]. Recently, $\mathrm{Wu}$ et al. [13] carried out MDF on the as-cast ZK21 Mg alloy and observed dissimilar microstructure for the samples forged at different strain rates. They observed an ultrafine DRX microstructure in the high strain rate-forged samples with an average grain size (AVG) of $0.3 \mathrm{~mm}$; whereas low strain rate-forged samples fail to obtain a complete DRX microstructure. Miura et al. [12] applied multidirectional forging to a $\mathrm{Mg}-\mathrm{Al}-\mathrm{Zn}$ alloy at room temperature and observed the suppression of the sharp textures due to twins in the microstructure, and equiaxed ultrafine grains after 20 forging passes.

Besides, recent reports [14-18] significantly exposed that twins refine the original coarse grains and serve as nucleation sites to promote an obvious DRX behavior [19] and are referred to as twin-induced DRX mechanism [14]. Therefore, to observe the deformation twins we exposed a (Mg-9.02Gd-4.21Y-0.48Zr wt\%) GW94 alloy by a small 
strain per pass and high-speed multidirectional impact forging (MDIF) process. As proposed before (MDIF) on commercial AZ61 alloy [14], in twin-induced DRX associated with $\{10-11\}$ contraction, and $\{10-11\}-\{10-12\}$ double twins, the coalescence and intersection of $\{10-12\}$ extension twins effectively refined the original coarse grains, contributing to the subsequent DRX process and formation of non-basal textures. Thus the new multidirectional impact forging (MDIF) technique with small strain per pass and high speed is proficient and attractive to produce bulk samples with a high density of twins and nonbasal texture.

In the previous report, MDIF was successfully applied to an Mg-Gd-Y alloy [20]. The workability was significantly improved and the MDIFed alloy exhibited superplastic behavior even at $400{ }^{\circ} \mathrm{C}$. However, the grain refinement mechanisms, especially twinning and DRX behavior, in GW94 alloy during MDIF and unidirectional impact forging (UDIF) are not clear at all in details.

Therefore in the present work, we focused on the microstructure evolution during the MDIF process with increasing forging passes. Besides, GW94 alloy was also subjected to unidirectional impact forging (UDIF) and our purpose was to clarify the differences in microstructure evolution, especially twinning and DRX behavior, during MDIF and UDIF and their contribution to the texture formation.

\section{Experimental Procedures}

GW94 (Mg-9.02Gd-4.21Y-0.48Zr wt\%) samples were machined in cubic block shape from the as-cast GW94 Mg ingot. All the cubic samples have dimensions of $(70 \mathrm{~mm} \times 70 \mathrm{~mm} \times 70 \mathrm{~mm})$. The samples were then solutionized at $510{ }^{\circ} \mathrm{C}$ for $6 \mathrm{~h}$ and finally cooled in air. The cubic samples were first heated to $450{ }^{\circ} \mathrm{C}$ in an electric resistance furnace and kept for $1 \mathrm{~h}$. Finally, the impact forging process was carried out using an industrial air pneumatic hammer machine with load gravity of $400 \mathrm{~kg}$. The MDIF plus UDIF are illustrated in detail in (Fig. 1). Briefly, during MDIF the forging direction was changed pass-by-pass $90^{\circ}$ (i.e., $\mathrm{X}$ to $\mathrm{Y}$ to $\mathrm{Z}$ to $\mathrm{X}$ and so on) as shown in (Fig. 1a); however, the forging direction was not changed during UDIF (Fig. 1b). The surface of the samples was kept smooth and regular so that the contact area between the sample and the load hammer was almost constant and the load was delivered to every point equally. Totally, six samples were impact forged, i.e., three samples were forged in one direction for 9, 29 and 50 passes and the other three samples were subjected to multidirectional 9, 29 and 50 passes and finally cooled down in air. They are denoted as UDIF-9, UDIF-29, UDIF-50 and MDIF-9,
MDIF-29 and MDIF-50, respectively. It was difficult to maintain the same strain per pass during the forging process; however, strain per pass $\sim 0.06$ was employed during MDIF and the average strain rate was around $20 \mathrm{~s}^{-1}$. For UDIF, almost the same force was applied at each forging pass, but the sample demonstrated a much more rapid strain hardening effect than that of MDIFed one; the pass strain quickly decreased with the increase of the forging passes. Beside this, the UDIF process took almost half the time as that taken by MDIF due to forging at one direction. The total strain of the UDIF-9, UDIF-29, and UDIF-50 samples was 33\%, 51\%, and 56\%, respectively, and the corresponding average pass strain was $0.03 \%$, $0.017 \%$, and $0.011 \%$. During the impact forging process, the sample temperature was maintained higher than $380{ }^{\circ} \mathrm{C}$ due to the rise in the temperature of the sample caused by high strain rate forging and limited heat emission for short forging time. These MDIFed and UDIFed samples were free from any surface defects as shown in Fig. 1c.

The microstructures were investigated at the central part of the last forging plane as shown in Fig. 1d by optical microscopy (OM), scanning electron microscopy (SEM), electron back-scatter diffraction (EBSD), and X-ray diffraction (XRD). For optical microscopy, the samples were etched with acetic picral ( $2 \mathrm{~g}$ of picric acid, $5 \mathrm{ml}$ of acetic acid, and $5 \mathrm{ml}$ of water, and $25 \mathrm{ml}$ of ethanol) for 4 to $5 \mathrm{~s}$. EBSD observation was carried out by using a Philips XL 30 ESEM- FEG/EDAX scanning electron microscope operating at $20 \mathrm{kV}$ and the corresponding probe current was $40 \mathrm{nA}$. Orientation imaging microscopy was measured at a step size of $0.2 \mu \mathrm{m}$. Pole figures were measured up to a tilt angle of $70^{\circ}$ using the Schultz reflection method by XRD. Defocusing corrections were made using experimentally determined defocusing curves from random powder samples.

\section{Results and Discussion}

\subsection{Initial Microstructure Before MDIF and UDIF}

Figure 2 shows the optical and SEM microstructures of the as-cast and as-solutionized GW94 alloy and their EDS analysis. The as-cast sample showed a dendritic eutectic microstructure consisting of an $\alpha-\mathrm{Mg}$ matrix and coarse eutectic $\beta$-phase mainly distributed along the grain boundaries as shown in Fig. 2a. Gao. et al. [21] have confirmed that the dendritic eutectic phase corresponded to the Mg24(Gd, Y)5 phases, which have a body-centered cubic (bcc) crystal structure with $a$ of $1.126 \mathrm{~nm}$. After solutionizing treatment at $510^{\circ} \mathrm{C}$ for $6 \mathrm{~h}$, the eutectic $\beta$ phase almost completely dissolved into the matrix, but still a few of cuboid-shaped particles remained throughout the 
(a)

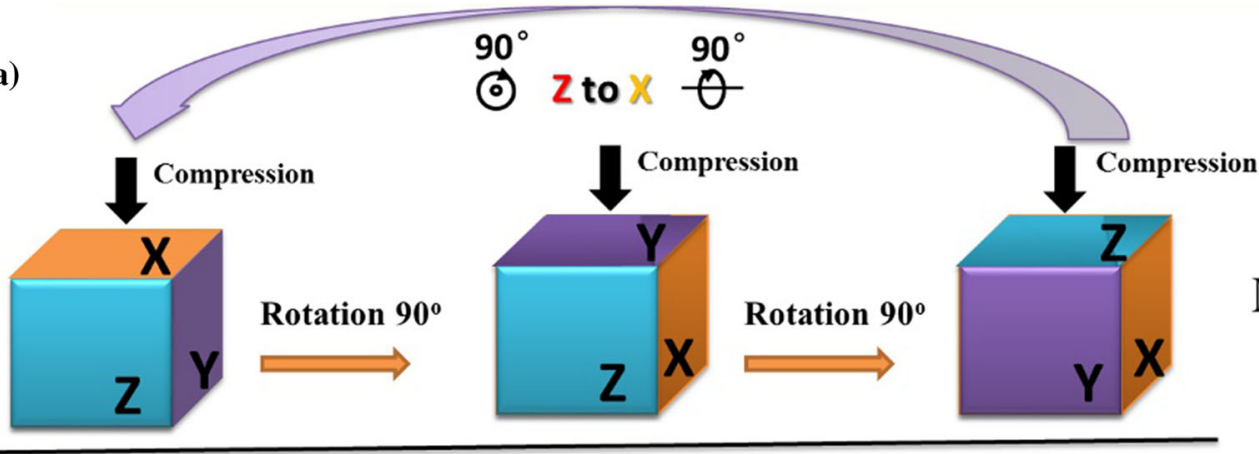

MDIF

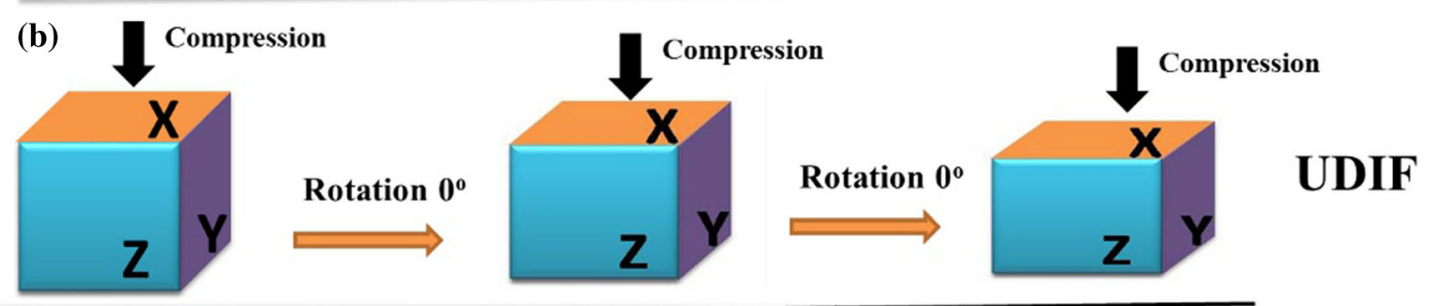

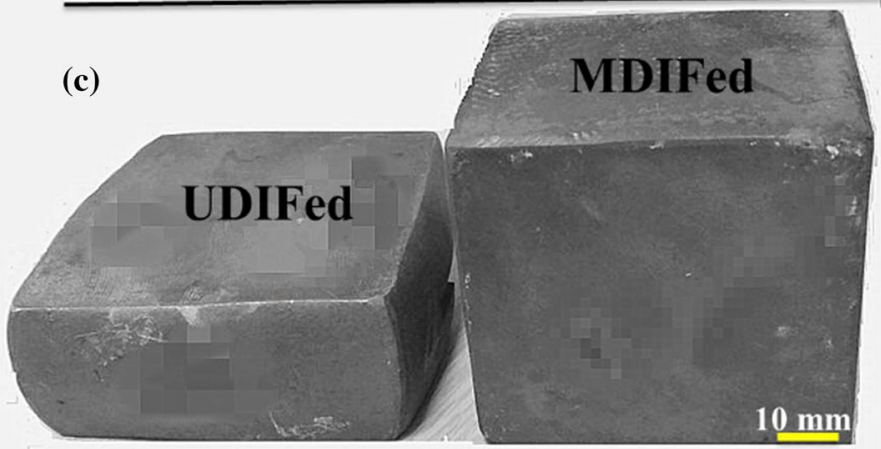

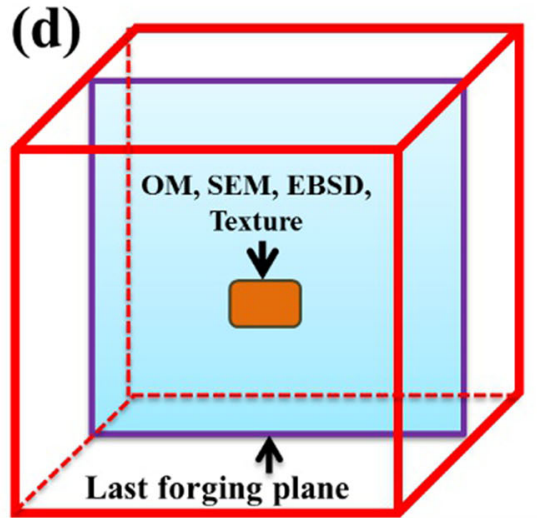

Fig. 1 Schematic illustrations of $\mathbf{a}, \mathbf{b}$ MDIF and UDIF processes, $\mathbf{c}$ macroscopic morphology of forged samples after 50 passes, $\mathbf{d}$ the position of microstructural and tensile samples machined out from the forged sample

solutionized microstructure as shown in Fig. 2f, which has also been identified as $\mathrm{Mg} 5(\mathrm{Gd}, \mathrm{Y})$ phases with fcc crystal structure having $a$ of $2.223 \mathrm{~nm}$ [21]. The average grain size of the as-solutionized GW94 alloy slightly grew up, from $\sim 85 \mu \mathrm{m}$ of the as-cast one to about $\sim 160 \mu \mathrm{m}$.

\subsection{Microstructure Evolution During Impact Forging}

Figure $3 \mathrm{a}-\mathrm{c}$ represents the typical optical microstructures of GW94 alloy during the MDIF process. After nine forging passes, plenty of twins were observed parallel or intersected with each other in some grains (Fig. 3a). The initial coarse grains were effectively divided into many fine separate thin parts by these twins. However, it should be noted that the twins were not observed in some grains as indicated by yellow arrows in Fig. 3a. As the forging passes increased from 9 to 29 , the amount of the twin matrix (TM) laminates decreased significantly and their sizes turn to be smaller as shown in Fig. 3b. In addition, DRX microstructure developed markedly, not only occupying most of the original GBs but also entering into the grain interiors. After 50 forging passes, a fully DRXed microstructure formed (Fig. 3c). An inspection of the microstructures in Fig. 3a-c revealed that the twins gradually disappeared and the fraction of equiaxed DRXed grains increased progressively with continuous deformation. After 50 forging passes, a homogenous microstructure with equiaxed grains was obtained, which indicates a completion of DRX process.

Figure $3 \mathrm{~d}-\mathrm{f}$ illustrates the optical microstructures of the UDIFed samples. After nine forging passes, the deformed grains seemed to extend along the direction normal to the compression direction and lots of elongated deformed grains appeared. Some fine parallel twins (seemingly) with each other could also be observed in some grains as indicated by red arrows in Fig. 3d. As the forging passes increased from 9 to 29 , the number of the twin laminate 


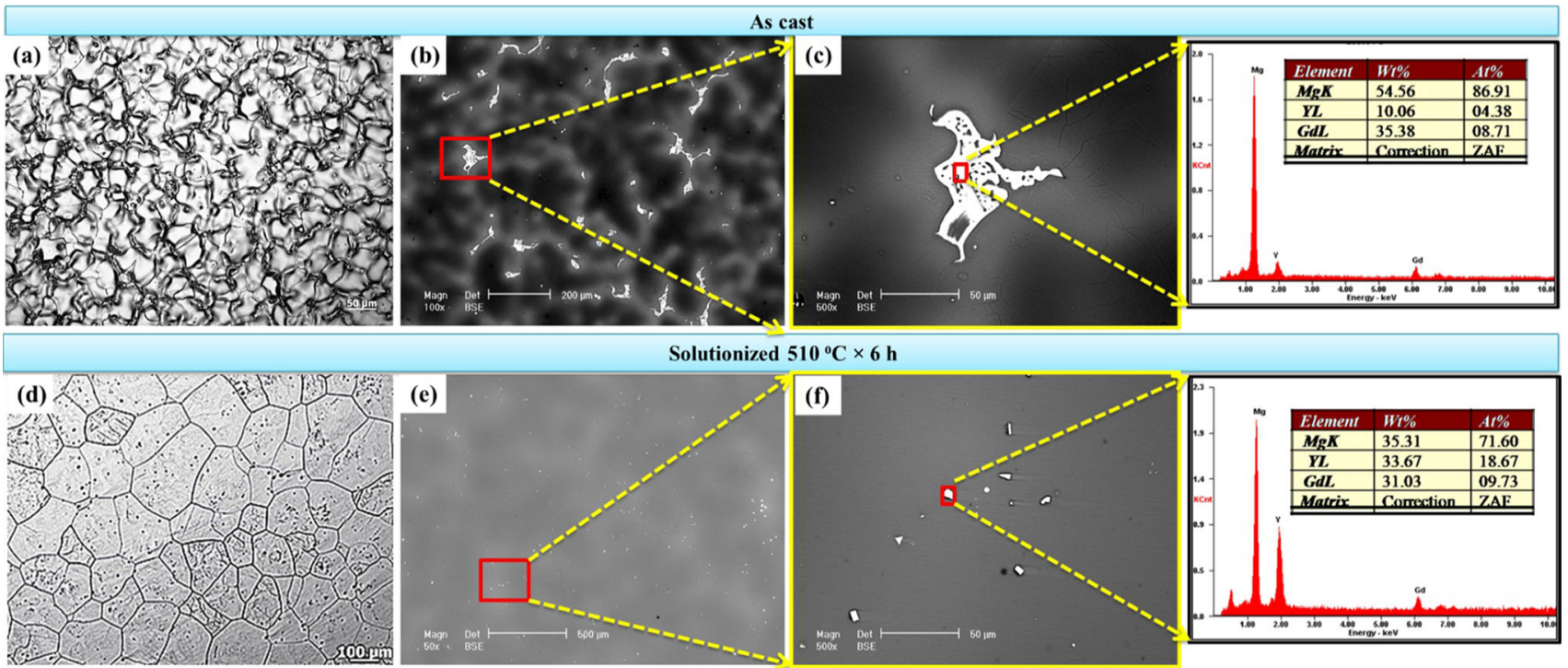

Fig. 2 Optical and SEM microstructures of GW94 alloy and their EDS analysis, respectively: a-c the as-cast, $\mathbf{d}-\mathbf{f}$ solutionized samples. EDS results of the inscribed areas selected in $\mathbf{c}, \mathbf{f}$

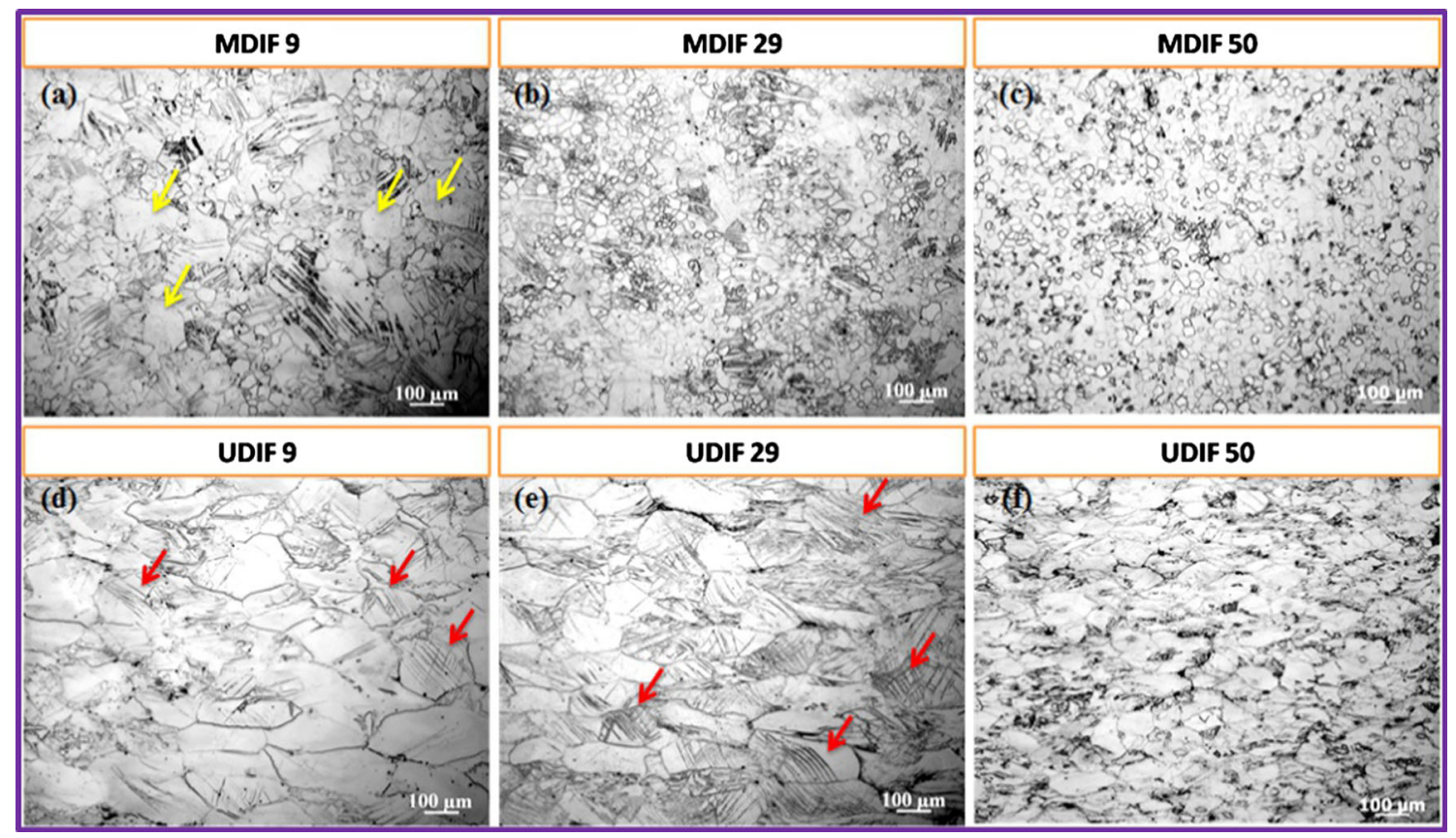

Fig. 3 Optical microstructure evolution of GW94 alloy with different forging passes: a MDIF9, b MDIF29, c MDIF50, d, UDIF9, e UDIF29, f UDIF50

increased significantly and turned to be more thinner (Fig. 3e). The grain refinements during the UDIF were not significant and the alloy seems not to obtain a fully recrystallized microstructure even after 50 passes (Fig. 3f).

To analyze the DRXed grains as well as the twins fraction, EBSD was performed on the MDIFed and UDIFed samples with different forging passes and the corresponding results are presented in Figs. 4 and 5. Low angle grain boundaries (LAGBs, $2^{\circ}-15^{\circ}$ ), high angle grain boundaries (HAGBs, $>15^{\circ}$ ) and twin boundaries, including $\{10-12\}$ extension, $\{10-11\}$ contraction and $\{10-11\}-$ $\{10-12\}$ double twin boundaries, are highlighted in different colors. The angular deviation from the twin boundaries was $5^{\circ}$ of the ideal value.

Figure $4 \mathrm{a}-\mathrm{f}$ represents the EBSD results after different MDIF passes. The IPF and the corresponding boundary misorientation maps reveal the conversion of the inhomogeneous microstructure that consisted of $\{10-12\}$ 


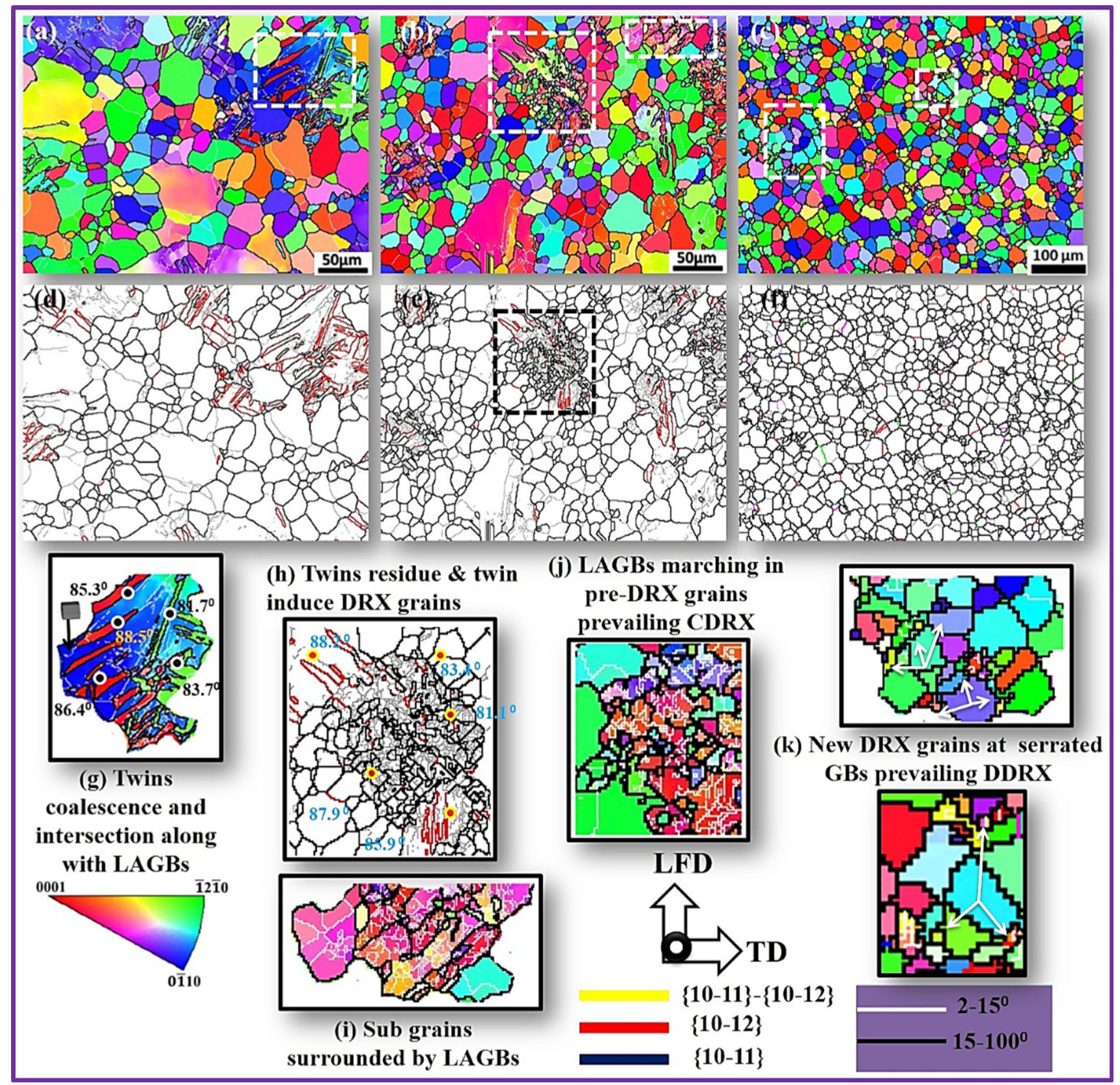

Fig. 4 The inverse pole figure (IPF) maps and corresponding boundary misorientations maps of the GW94 alloy after MDIF process: a, d MDIF9, b, e MDIF29, c, f MDIF50. Here, $\mathbf{g}-\mathbf{k}$ are the enlarged IPF maps selected in $\mathbf{a}, \mathbf{b}$, $\mathbf{c}$, e, respectively, showing the twins and the formation of sub-GBs and DRXed grains by CDRX

extension twins, and relatively coarse grains into a fine homogeneous microstructure. To understand the microstructure evolution in detail, different typical regions were selected (Fig. 4a-f) and magnified (Fig. 4g-k). After nine passes, nearly all the twins activated were $\{10-12\}$ extension twins. The presence of $\{10-12\}$ extension twins could be attributed to their low critical resolved shear stress (CRSS) in the range of 2-3 MPa [22, 23]. Careful observation revealed the intersections of the twins in some particular regions (Fig. 4g) which could be shown more clearly in Fig. 4h, providing an apparent proof for twin induce DRX mechanism; i.e., the rearrangement of dislocations within the twins further subdivided it into nonequilibrium sub-grains, and then converted into the grains with HAGBs with further increase in strain [13, 24]. Moreover, the intersection of those twin boundaries can demolish their coherency and supply a large density of dislocations, thus offer a larger driving force than the parallel twins, suggesting more effective nucleation sites for recrystallization $[25,26]$. Besides the parallel or 

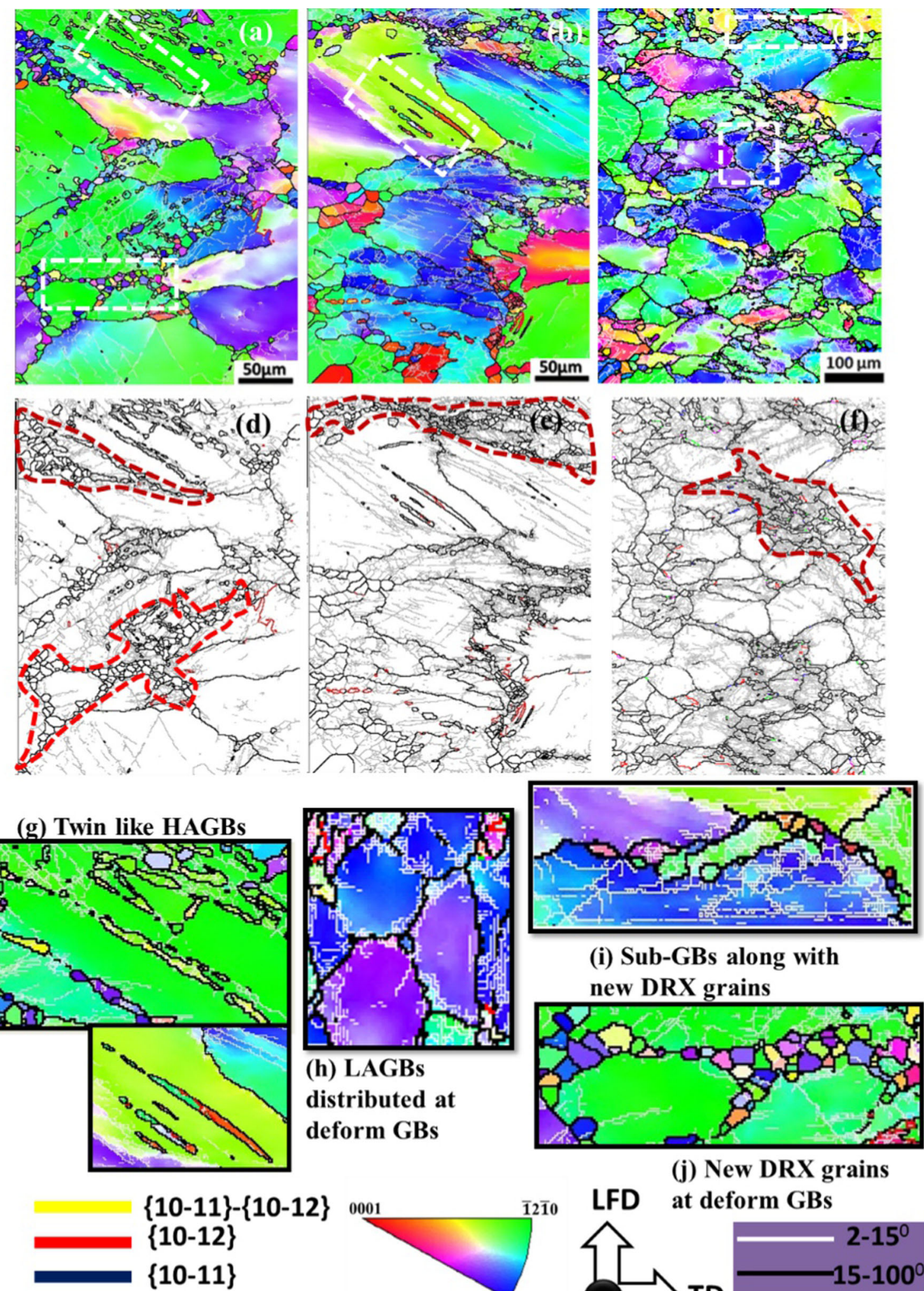

(i) Sub-GBs along with new DRX grains

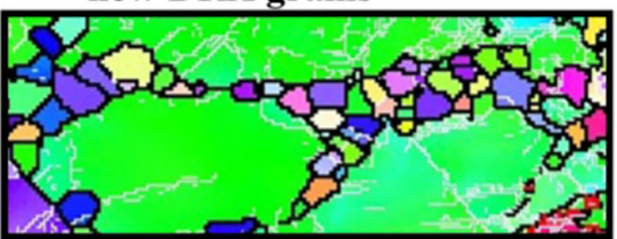

(j) New DRX grains

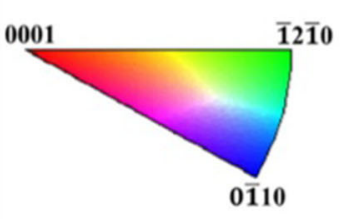

LFD at deform GBs

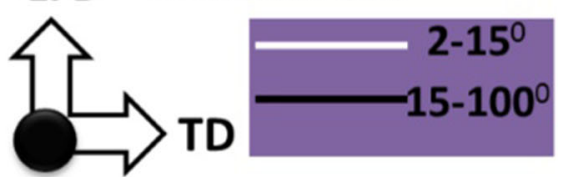

Fig. 5 The inverse pole figure (IPF) maps and corresponding boundary misorientation maps of the GW94 alloy after UDIF process: a, b UDIF9, $\mathbf{c}, \mathbf{d}$ UDIF29, e, f UDIF50. Here, $\mathbf{g}-\mathbf{i}$ are the enlarged IPF maps selected in $\mathbf{a}, \mathbf{b}, \mathbf{c}$, respectively, showing the formation of sub-GBs along deform GBs and DRXed grains 
intersected $\{10-12\}$ extension twins, the internal stresses may also tend to be relieved by the rearrangements of dislocations during the recovery process, which finally results in the formation of LAGBs [27]. Some mechanisms for the formation of LAGBs leading to sub-grain boundary migration (SBM) have been suggested before in Mg alloys [28] that accordingly give rise to the new grains with HAGBs. Similarly in the present case, a lot of the LAGBs were observed. Some of the LAGBs tend to form subgrains (Fig. 4i), while the others are marched into the grain interiors by a continuous DRX behavior (Fig. $4 \mathrm{j}$ ), which is a recovery-dominated process and proceeds by a continuous absorption of dislocations in sub-GBs, and finally gives rise to the formation of new DRXed grains (Fig. 4j).

Eventually, when the sample was forged for 50 passes, the DRX consumed most of the $\{10-12\}$ extension twins. Small new DRXed grains (2-3 um) were formed at the original GBs and their orientations were found to be much different from their parent's grains as indicated in the IPF map (Fig. 4k). This suggests that a new DRX mechanism took place; i.e., with continuous deformation, the previous DRXed grains formed by continuous DRX were further refined through a discontinuous DRX process, which is a conventional DRX process with nucleation and bulging of LAGBs at serrated HAGBs [29-31]. The formation of such a small grains $(<5 \mathrm{um})$ besides the serrated GBs of relatively large DRXed grains was observed [14]. Thus, a homogeneous microstructure with very few of LAGBs and almost no twins was obtained, which entails a completion of DRX process.

Figure 5 represents the IPF maps during the UDIF process. Figure $5 \mathrm{a}$ and $\mathrm{b}$ exhibits quite similar inhomogeneous deform microstructure. The microstructures of the samples contain two distinct features. The elongated grains are distributed in the regions, which lie nearly perpendicular to the forging direction as shown in Fig. 5a and b, whereas equiaxed small grains with HAGBs are distributed along the deformed grain boundaries as inscribed by red lines in the corresponding boundary misorientation maps. With the increase of the total strain, almost from 9 to 50 forging passes, instead of twinning, the UDIF process was dominated by dislocation slips, which were exposed by a large number of LAGBs [27].

To understand the microstructure evolution in detail, different typical regions were selected in Fig. 5a-f and are magnified in Fig. $5 \mathrm{~g}-\mathrm{j}$. Apparently, at the initial stage of UDIF process, the twin-like structures were observed in Fig. 5g; however, they were not marked as twin boundaries during the EBSD analysis. It may be that the former twins occurred before nine forging passes and tended to disappear, either due to the de-twinning behavior or their deviation from the ideal twin rotation axis, as in the study of 1-Samman et al. [28] of the twin-induced DRX behavior during $c$-axis compression in $\mathrm{Mg}$ single crystal. They found that the rotation axis of the new GBs separating DRXed grains from the matrix could deviate from the ideal twin rotation axis, $<1-210>$ within a maximum range of $30^{\circ}$. As a result, those special GBs were not detected as twin boundaries during the EBSD analysis (Fig. $5 \mathrm{~g}$ ). On the other hand, almost no twins were observed during the further UDIF process even up to 50 forging passes. The reason may be that the twinning activation in $\mathrm{Mg}$ is dependent on applied stress and grain orientation. Unidirectional compression accumulates the basal orientations to the compression direction [32]; i.e., the basal plane of the grains with $c$-axis almost parallel to the forging direction and results in a strong basal texture (Fig. 6), which is most suitable for the activation of $\{10-11\}$ contraction twins. However, the higher CRSS value for $\{10-11\}$ contraction twin than the basal/non-basal slips leads to the difficulty for their nucleation. Indeed the CRSS of $\{10-12\}$ extension twins are comparable to the CRSS of some slips, but due to unsuitable grain orientations they cannot be expected to happen. Additionally, the temperature drop during the deformation also has a significant impact on twinning and slip behavior. It is commonly known that the CRSS for non-basal slip system considerably decreases at higher temperature [14]. Though the forging process was carried out at room temperature, and the temperature dropped sharply, the temperature of the sample subjected to UDIFed 50 passes was $\sim 442{ }^{\circ} \mathrm{C}$, much greater than the $\sim 380{ }^{\circ} \mathrm{C}$ for MDIFed one. Indeed, the samples before MDIF and UDIF were heated for the same temperature at $450{ }^{\circ} \mathrm{C}$ for $\sim 60 \mathrm{~min}$, but changing the direction of the sample during the MDIF process consumes time, whereas forging only at one direction in UDIF process takes much lesser time and thus results in a less temperature drop. The additional factor such as strain hardening effect [33] in UDIFed sample may also keep the high temperature during the deformation process. Thus in the present case $\left(442{ }^{\circ} \mathrm{C}\right.$ our experiment), the increased temperature stimulated the dislocation slips on basal and non-basal planes instead of high CRSS $\{10-11\}$ contraction twinning.

Moreover, it is claimed that the grains with the basal texture are relatively stable and insensitive to further DRX [34], and the basal/non-basal cross-slips tend to occur in highly strained regions such as grain boundaries [35]. The interaction of those basal/non-basal cross-slips not only assist the formation of dislocation, but also yield LAGBs (Fig. 5h) and sub-GBs (Fig. 5i), respectively [30, 36]. Consequently, the formation of those sub-grains progressively guides to the development of new DRXed grains (Fig. 5j) [37]. Therefore, we suggest that the observed mantle or (necklace) type DRX grains settled near the grain boundaries appeared due to the ingestion of those of dislocations to sub-GBs, which finally give rise to the 


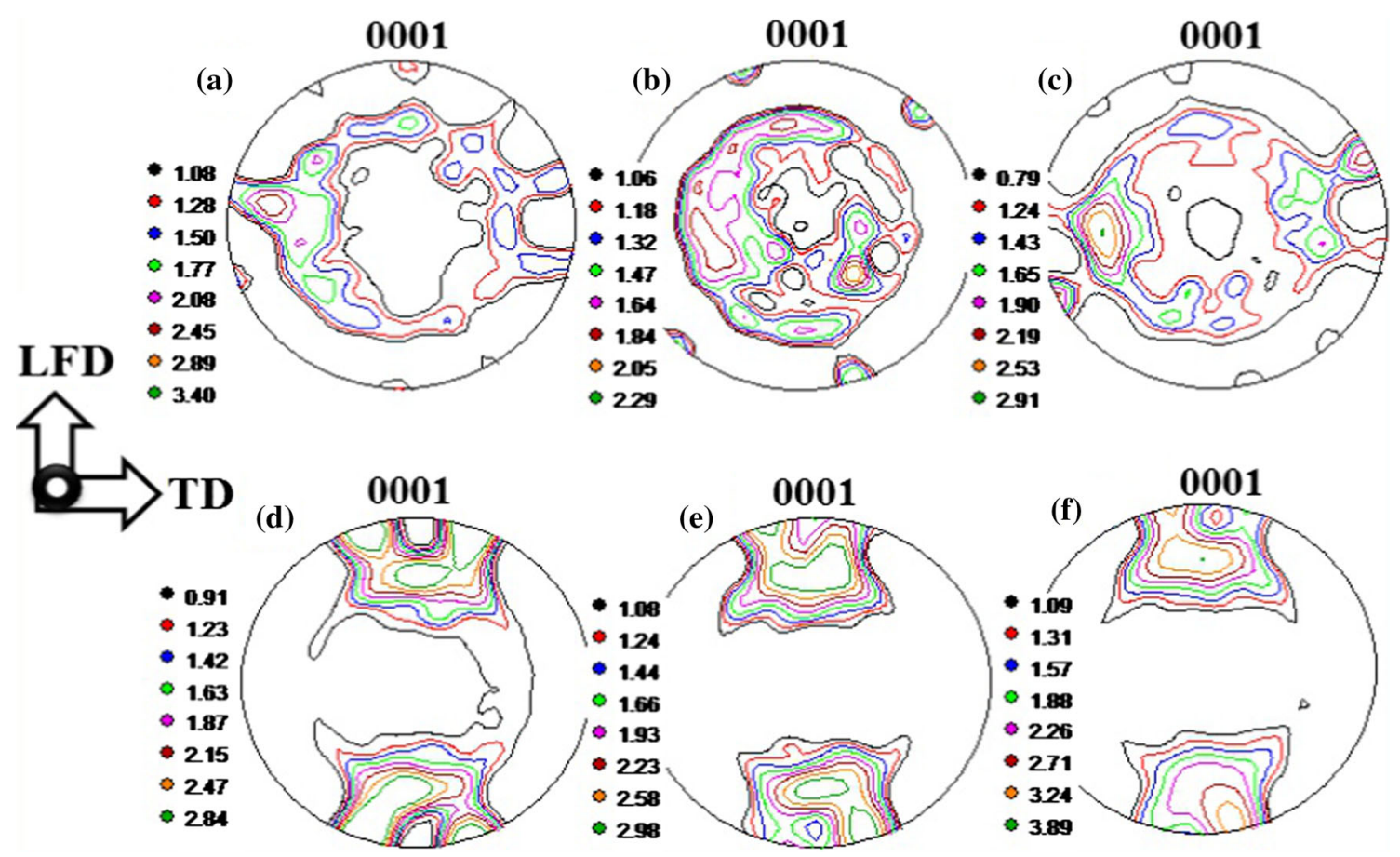

Fig. 6 Obtained pole figures of the GW94 alloy after MDIF and UDIF process which reveal in sequence as a-c represent the MDIF9, MDIF29, and MDIF50 samples, respectively, whereas d-f represent UDIF9, UDIF29, and UDIF50 samples, respectively

necklace-type structure in order to soften the material [38]. This "necklace-type structure" could be attributed to the discontinuous dynamic recrystallization (DDRX) at this stage [38] despite the fact that a large amount of LAGBs symbolized for continuous dynamic recrystallization (CDRX) could be observed; however, their extent increases throughout the microstructure and remained until 50 passes (Fig. 5c). This suggests that UDIF is relatively slow to obtain a homogeneous microstructure in comparison to MDIF. The DRXed grain sizes did not change drastically, but their fraction increased slightly during the UDIF process.

Figure 6 shows the $\{0001\}$ pole figures of the MDIFed and UDIFed samples. The MDIFed sample exhibited a non-basal and almost similar texture under different passes, which could be ascribed to the increase in volume fraction of DRXed grains and mainly to the multidirectional impact forging process and its characteristics as reported previously that MDF support to randomize the texture $[39,40]$. Moreover, as a good amount of $\{10-12\}$ extension were induced during the MDIF process, and it is well known that the activation of twins depends on the relative orientation of $c$-axes of the crystallites and the applied stress. $\{10-12\}$ extension twins could be activated when the forging direction is perpendicular to $c$-axis, while $\{10-11\}$ contraction twins occur under the compression along $c$-axis. Therefore, the continuous change in the forging direction would activate the twins with different orientation in original coarse grains as they have a different orientation (as-solutionized condition). Also, $\{10-12\}$ extension twins change the basal plane of the grains with $c$-axis perpendicular to the forging direction by $\sim 86^{\circ}$, i.e., $c$-axis almost parallel to the last forging direction. Therefore, based on a randomly oriented parent grains at the initial stage of the forging process, randomly oriented twins resulted in the non-basal texture during the MDIF process. The random orientation of the grains were kept and continued following the DRX process, and thus a weak texture was maintained during the MDIF process. However, the UDIFed sample showed a strong basal texture. As mentioned above, unidirectional compression accumulates the basal orientations parallel to the compressive plane, which in turn yielded out the dominant basal texture [32]. Although the addition of rare earth elements can significantly modify the traditional texture which concentrates around the $\{0001\}$ plane even at very low concentrations in $\mathrm{Mg}$ alloys [41-44], a strong basal texture was obtained in our case of UDIF, which brings us to suggest that deformation technique such as unidirectional forging has a strong effect on the final texture of the sample even though a large amount of RE elements were present.

In addition, most researchers believed that the effect of $\mathrm{RE}$ additions on texture modification is principally focused on the recrystallization behavior; Shilun [45], Li [1] and Yan et al. [46] have also reported that the DRXed grains has an important role in the weakening of overall texture by 
counteracting the strong deformation texture of the parent grains. This is consistent with the present results; i.e., the UDIFed deform grains correspond to the strong basal texture (Fig. 6), while the mature DRXed grains deviate from the basal pole in MDIFed samples. Therefore, the MDIF process is a convenient way to obtain randomized texture in GW94 alloy.

\subsection{Different Microstructure During MDIF and UDIF}

As compared to the MDIFed sample at different passes, the UDIFed sample shows quite a different developed microstructure. The reason may be that the absence of twins induces DRX in UDIFed samples, whereas $\{10-12\}$ extension twins were vigorous in promoting DRX during the MDIF process.

Twinning in hexagonal close packing (hcp) materials tends to recompense the limited effective slip systems by reorienting the grains in other direction to re-activate those slip systems or accommodate the strain itself. In contrast, dynamic recrystallization (DRX) is a process of restoration or softening mechanism, which facilitate the grain refinement process [15]. Thus, twinning and DRX are both important for plastic deformation of magnesium alloys.

It is evident that during the MDIF the original coarse grains were not only divided by a large number of $\{10-12\}$ extension twins, but also contributed to the DRX during MDIF as observed before in detail (Fig. 4). However, in the regions without those twins in UDIFed samples, DRX was harder to be initiated so that deform grains surrounded by regions with fine DRXed grains were observed (Fig. 5). In the deformed grains, a large amount of LAGBs (precursor of dislocations) were also observed (Fig. 5). Though the grain boundaries and twins could both be the nucleation sites for DRX, the DRX on twins seem to be more efficient than the DRX on the grain boundaries during the microstructure evolution. It is well known [47] that five independent plastic deformation modes are essential to accommodate the plastic deformation in polycrystalline HCP metals. While the crystallographic slips change the lattice orientation of material gradually, deformation twinning changes it abruptly and offers an additional mode to accommodate strain [24]. Therefore, during the high strain rate MDIF process, twinning performs an essential role than that by dislocation glides, since appropriate slip systems could not be activated as instantly as twins [18]. Regarding the role of twins on DRX, Ma et al. [15] pointed out the effect of twinning and dynamic recrystallization on the high strain rate rolling process (HSRR) of ZK60 Mg alloy and show that the DRX on twins is more important than the DRX on grain boundaries during high strain rate rolling (HSRR). Zhu et al. [18] investigated the microstructural evolution of a ZK60 Mg alloy during the high strain rate rolling process and proposed that a large number of twins are responsible for fully DRXed microstructure. Indeed, contraction and double twins are thought to be more efficient during recrystallization than the $\{10-12\}$ extension twins $[25,26]$; however, $\{10-12\}$ extension twins still can possess an active role during the deformation process as observed in our previous experiments [9] and also as reported before by Ma et al. [15].

Therefore in the case of the sample, i.e., MDIFed displays homogeneous microstructures in comparison to UDIFed, which could be attributed to the beneficial twin inducing DRX nucleation during the MDIF process.

\section{Conclusions}

In summary, multi- and unidirectional impact forging was successfully applied to bulk Mg-RE samples. The DRX and grain refinement mechanisms were investigated and discussed in detail. The main results are described below:

1. MDIF is an effective method to optimize the grain size, texture and morphology. In contrast to UDIF, it is much quicker to obtain a homogeneous microstructure in Mg-RE alloys.

2. During the UDIF, the dislocation slips in the neighborhood of the grain boundaries reorder themselves to form a new group of LAGBs, which in turn leads to the formation of sub-grains, and then the conversion of these sub-grains with LAGBs to HAGBs occurs, hence ensuring a bimodal chain microstructure.

3. A homogeneous microstructure with an AGS of $15.5 \mu \mathrm{m}$ was obtained from the coarse original grains of $\sim 160 \mu \mathrm{m}$ after the MDIF process. This significant grain refinement is credited to consecutive twinning, DRX mechanism i.e., CDRX and DDRX at last stages, thus finally leading to almost a fully recrystallized microstructure. The cooperative effects of continuous DRX, twinning, and MDIF mainly contributed to the formation of special non-basal textures during MDIF, while in uniaxial forging, although the new DRX grains diverge the orientation from the strong basal plane, the UDIF still yielded the dominant basal texture due to their small volume fraction.

Acknowledgements This work was financially supported by the National Key Research and Development Program of China (No. 2016YFB0301104), the National Natural Science Foundation of China (Nos. 51531002, 51301173 and 51601193), and the National Basic Research Program of China (“973 Program”, No. 2013CB632202). 


\section{References}

[1] L. Li, Mater. Sci. Eng., A 528, 7178 (2011)

[2] T. Peng, Q. Wang, J. Lin, M. Liu, H.J. Roven, Mater. Sci. Eng., A 528, 1143 (2011)

[3] M. Hong, S. Shah, D. Wu, R. Chen, X. Du, N. Hu, Y. Zhang, Met. Mater. Int. 22, 1091 (2016)

[4] D. Yin, Q. Wang, C. Boehlert, V. Janik, Metall. Mater. Trans. A 43, 3338 (2012)

[5] D. Yin, Q. Wang, C. Boehlert, V. Janik, Y. Gao, W. Ding, Mater. Sci. Eng., A 546, 239 (2012)

[6] T. Homma, N. Kunito, S. Kamado, Scr. Mater. 61, 644 (2009)

[7] C. Xu, S. Xu, M. Zheng, K. Wu, E. Wang, S. Kamado, G. Wang, X. Lv, J. Alloys Compd. 524, 46 (2012)

[8] C. Xu, M. Zheng, S. Xu, K. Wu, E. Wang, S. Kamado, G. Wang, X. Lv, Mater. Sci. Eng., A 547, 93 (2012)

[9] S.S.A. Shah, D. Wu, W.H. Wang, R.S. Chen, Mater. Sci. Eng., A 702, 153 (2017)

[10] A. Belyakov, T. Sakai, H. Miura, Mater. Trans., JIM 41, 476 (2000)

[11] O. Sitdikov, T. Sakai, H. Miura, C. Hama, Mater. Sci. Eng., A 516, 180 (2009)

[12] H. Miura, T. Maruoka, X. Yang, J. Jonas, Scr. Mater. 66, 49 (2012)

[13] Y.Z. Wu, H.G. Yan, J.H. Chen, Y.G. Du, S.Q. Zhu, B. Su, Mater. Sci. Eng., A 556, 164-169 (2012)

[14] M. Jiang, H. Yan, R. Chen, J. Alloys Compd. 650, 399 (2015)

[15] Q. Ma, B. Li, E.B. Marin, S.J. Horstemeyer, Scr. Mater. 65, 823 (2011)

[16] S. Shah, D. Wu, W. Wang, R. Chen, Mater. Sci. Eng., A 702, 153 (2017)

[17] S. Zhu, H. Yan, X. Liao, S. Moody, G. Sha, Y. Wu, S. Ringer, Acta Mater. 82, 344 (2015)

[18] S.Q. Zhu, H.G. Yan, J.H. Chen, Y.Z. Wu, J.Z. Liu, J. Tian, Scr. Mater. 63, 985 (2010)

[19] L. Tang, Y. Zhao, N. Liang, R. Islamgaliev, R. Valiev, Y. Zhu, Mater. Sci. Eng., A 677, 68 (2016)

[20] J. Li, D. Wu, Q. Yang, R. Chen, J. Alloys Compd. 672, 27 (2016)

[21] L. Gao, R. Chen, E. Han, Mater. Sci. 44, 4443 (2009)
[22] A. Kaya, Fundam. Magnes. Alloy Metall. 33, 35 (2013)

[23] M. Barnett, Z. Keshavarz, X. Ma, Metall. Mater. Trans. A 37, 2283 (2006)

[24] J.W. Christian, S. Mahajan, Prog. Mater Sci. 39, 1 (1995)

[25] L. Lu, J. Zhao, L. Liu, G. Wang, Mater. Sci. Technol. 32, 104 (2016)

[26] I. Basu, T. Al-Samman, Acta Mater. 96, 111 (2015)

[27] W.D. Callister Jr., D.G. Rethwisch, Fundamentals of Materials Science and Engineering: An Integrated Approach (John Wiley \& Sons, Hoboken, 2012)

[28] T. Al-Samman, K.D. Molodov, D.A. Molodov, G. Gottstein, S. Suwas, Acta Mater. 60, 537 (2012)

[29] T. Al-Samman, G. Gottstein, Mater. Sci. Eng., A 490, 411 (2008)

[30] O. Sitdikov, R. Kaibyshev, Mater. Trans. 42, 1928 (2001)

[31] C.H. Park, C.S. Oh, S. Kim, Mater. Sci. Eng., A 542, 127 (2012)

[32] X.-Y. Yang, Z.S. Ji, H. Miura, T. Sakai, Chin. J. Nonferr. Metal. 19, 55 (2009)

[33] E. Martín, J. Mater. Process. Technol. 143, 1 (2003)

[34] Q. Jin, S.Y. Shim, S.G. Lim, Scr. Mater. 55, 843 (2006)

[35] J.J. Jonas, S. Mu, T. Al-Samman, G. Gottstein, L. Jiang, Ė. Martin, Acta Mater. 59, 2046 (2011)

[36] J. Koike, T. Kobayashi, T. Mukai, H. Watanabe, M. Suzuki, K. Maruyama, K. Higashi, Acta Mater. 51, 2055 (2003)

[37] M. Wang, R. Xin, B. Wang, Q. Liu, Mater. Sci. Eng., A 528, $2941(2011)$

[38] L. Tang, C. Liu, Z. Chen, D. Ji, H. Xiao, Mater. Des. 50, 587 (2013)

[39] X.Y. Yang, Z.Y. Sun, X. Jie, H. Miura, T. Sakai, Chin. J. Nonferr Metal. 18, s200 (2008)

[40] D. Wu, R. Chen, W. Tang, E. Han, Mater. Des. 41, 306 (2012)

[41] L. Mackenzie, M. Pekguleryuz, Scr. Mater. 59, 665 (2008)

[42] N. Stanford, M. Barnett, Mater. Sci. Eng., A 496, 399 (2008)

[43] N. Stanford, Mater. Sci. Eng., A 527, 2669 (2010)

[44] N. Stanford, M. Barnett, Scr. Mater. 58, 179 (2008)

[45] Y. Shilun, G. Yonghao, L. Chuming, X. Hongchao, Mater. Sci. Forum 849, 181 (2016)

[46] H. Yan, R. Chen, E. Han, Mater. Charact. 62, 321 (2011)

[47] R. Von Mises, Appl. Math. Mech. 592, 8 (1928) 